\title{
Asente Antifator de Necrose Tumoral $\alpha$ no Tratamento da Artrite Reumatóide na Prática Clínica Diária
}

\section{Anti-Tumor Necrosis Factor $\alpha$ Asent in the Treatment of Rheumatoid Arthritis in the Clinical Practice}

\author{
Cristiane Kayser ${ }^{(1)}$, Alexandre Wagner Silva de Souza ${ }^{(1)}$
}

\begin{abstract}
A artrite reumatóide (AR) é uma doença inflamatória crônica na qual citocinas pró-inflamatórias, como o fator de necrose tumoral alfa (TNF), parecem exercer um papel central na patogênese desta. Agentes biológicos que inibem o TNF têm sido considerados eficazes na redução da atividade e no retardamento do dano estrutural articular na artrite reumatóide, em especial nas formas refratárias aos tratamentos convencionais. Atualmente, três agentes anti-TNF encontram-se disponíveis para o tratamento da AR: infliximabe (um anticorpo anti-TNF monoclonal quimérico), adalimumabe (anticorpo anti-TNF monoclonal humano) e etanercepte (receptor de TNF que age como inibidor competitivo do TNF- $\alpha$ e do $\beta$ ). Entretanto, estudos mais recentes têm apontado para o fato de que as elevadas taxas de resposta descritas em ensaios clínicos randomizados geralmente não são encontradas na prática clínica diária. Este dado é de fundamental relevância para o reumatologista e deve ser levado em consideração quando do uso dessas drogas no consultório. Selecionamos aqui alguns artigos publicados recentemente que abordam o uso de agentes anti-TNF na prática clínica diária.
\end{abstract}

Kievit W, Fransen J, Oerlemans AJ, et al.: The efficacy of anti-TNF in rheumatoid arthritis, a comparison between randomized controlled trials and clinical practice (Eficácia dos anti-TNF na artrite reumatóide; comparação entre ensaios randomizados e controlados e a prática clínica). Ann Rheum Dis published online 10 Apr 2007. Radboud University Nijmegen Medical Centre, Netherlands.

Este estudo teve como objetivo comparar a eficácia dos agentes anti-TNF observada em ensaios controlados e randomizados com a eficácia encontrada em um estudo de coorte realizado na Holanda, que refletiria a prática clínica diária. Inicialmente, realizou-se uma revisão sistemática para a seleção de ensaios clínicos randomizados placebocontrolados escritos em inglês, e os artigos mais relevantes foram selecionados. Utilizaram-se para comparação os dados do Estudo DREAM (Dutch Rheumatoid Arthritis Monitoring). Este é um registro prospectivo realizado em 11 hospitais da Holanda, no qual são incluídos todos os pacientes que iniciaram o tratamento com agentes anti-TNF pela primeira vez. As seguintes variáveis foram comparadas: características dos pacientes, doses das medicações utiliza- das e taxa de resposta ACR20. As variáveis foram também estratificadas para a elegibilidade do paciente para participar ou não do ensaio clínico controlado. Em relação aos ensaios clínicos, foram selecionados 12 artigos que avaliaram o tratamento da AR com infliximabe $(\mathrm{n}=2)$, etanercepte $(\mathrm{n}=5)$ ou adalimumabe $(\mathrm{n}=5)$ versus placebo. Entre o grupo de pacientes do estudo DREAM, 546 pacientes foram incluídos para a análise. Cinco grupos de diferentes tratamentos foram observados: adalimumabe com metotrexato; monoterapia com adalimumabe; etanercepte com MTX; monoterapia com etanercepte; e infliximabe com MTX. Apenas pequenas diferenças foram observadas em relação às características clínicas dos pacientes do Estudo DREAM em comparação aos ensaios clínicos no baseline.

1. Médico(a)-assistente doutor(a) da Disciplina de Reumatologia da Escola Paulista de Medicina/Universidade Federal de São Paulo (EPM-Unifesp) Endereço para correspondência: Rua Botucatu, 740 - 3o andar, CEP 04023-062, São Paulo, SP, Tel.: (11) 5576-4239, e-mails: criskayser@terra.com.br; alexandre_wagner@uol.com.br. 
As doses de anti-TNF e MTX, assim como as de antiinflamatórios não hormonais utilizadas foram comparáveis. Vinte e nove a $54 \%$ dos pacientes do estudo DREAM estavam em uso de corticosteróides no início do estudo contra $44 \%$ e $69 \%$ nos ensaios clínicos, respectivamente. Em 10 de 11 comparações realizadas, a taxa de resposta ACR 20 foi menor no Estudo DREAM que nos ensaios clínicos controlados, e tal diferença foi estatisticamente significante em cinco das comparações. Apenas 34\% a $79 \%$ dos pacientes do Estudo DREAM preencheram os critérios de inclusão para doença ativa utilizados nos diferentes ensaios clínicos. Os pacientes do Estudo DREAM elegíveis para os ensaios clínicos obtiveram maior taxa de resposta ACR 20 do que os pacientes considerados inelegíveis. A taxa de resposta ACR 20 dos pacientes elegíveis foi semelhante à percentagem de resposta dos grupos de pacientes em uso de droga ativa nos ensaios clínicos em 10 das 11 comparações realizadas. Os autores concluem que a eficácia dos agentes anti-TNF foi superior nos ensaios clínicos controlados e randomizados. Entretanto, enfatizam que os pacientes tratados com agentes anti-TNF no Estudo DREAM possuíam, em geral, doença menos ativa quando comparados com os pacientes incluídos nos outros estudos e que, quando os pacientes do Estudo DREAM eram elegíveis para inclusão nos diferentes ensaios clínicos, a resposta ao tratamento passava a ser semelhante.

Zink A, Strangfeld A, Schneider M, et al.: Effectiveness of tumor necrosis factor inbibitors in rheumatoid arthritis in an observational cohort study: comparison of patients according to their eligibility for major randomized clinical trials (Efetividade dos inibidores do fator de necrose tumoral na artrite reumatóide em um estudo de coorte observacional: comparação de acordo com a elegibilidade dos pacientes para estudos clínicos randomizados). Arthritis Rheum 54(11): 3399-407, 2006. German Rheumatism Research Centre and Charité University Medicine, Berlin, Germany.

Neste estudo, os autores comentam que os resultados obtidos em ensaios clínicos randomizados (ECRs) para avaliar a eficácia terapêutica de uma determinada droga podem ser artificiais em virtude da utilização de critérios de inclusão e exclusão restritos, regimes de tratamentos fixos e acompanhamento rígido. Comentam ainda que estudos de coorte observacionais são importantes para complementarem os conhecimentos obtidos com os ECRs, pois incluem um amplo grupo de pacientes com várias comorbidades, uso concomitante de vários medicamentos, diferentes regimes terapêuticos e diversos estágios da doença. Com isso em mente, os autores utilizaram os dados de um registro germânico de pacientes com AR (RABBIT) que iniciaram o tratamento com agentes antiTNF (infliximabe, etanercepte ou adalimumabe) no período de 2001 a 2004, para avaliar: quantos desses pacientes seriam elegíveis para os principais ECRs que resultaram na aprovação dessas drogas para o tratamento da AR; qual é a resposta terapêutica dos pacientes considerados elegíveis e inelegíveis, utilizando o critério de resposta do Colégio Americano de Reumatologia (ACR 20 e ACR50), após 6 meses de acompanhamento. Um total de 1.458 pacientes completaram os 6 meses de acompanhamento do registro RABBIT e foram incluídos nas análises. Em relação aos resultados, apenas $21 \%$ a $33 \%$ dos pacientes do registro RABBIT seriam elegíveis para participarem dos principais
ECRs. Nesses pacientes, as respostas ACR20 e ACR50 foram comparáveis com as taxas de resposta encontradas nos diferentes ECRs, e a resposta ACR20 variou de $52 \%$ a $74 \%$ e a resposta ACR50, de $26 \%$ a $41 \%$. As taxas de resposta ACR foram inferiores naqueles pacientes considerados inelegíveis para os ECRs (resposta ACR20 de $44 \%$ a $57 \%$ e ACR50 de $19 \%$ a $25 \%$ ); entretanto, a resposta absoluta foi similar aos pacientes elegíveis. Os pacientes inelegíveis apresentavam menor atividade de doença no início do estudo, mais comorbidades e menor classe funcional. $\mathrm{Na}$ discussão, os autores comentam que os resultados obtidos nos ECRs refletem os dados de uma pequena proporção dos pacientes com AR tratados na prática clínica diária. $\mathrm{Na}$ "vida real", os pacientes com AR muitas vezes possuem estágio de doença mais avançado e diversas comorbidades, mas também necessitam de tratamento adequado. Além disso, na prática clínica, um índice de atividade elevado não é o único fator utilizado para a decisão terapêutica, e outros fatores incluindo falha terapêutica prévia, progressão radiológica e a condição individual do paciente são utilizados. No entanto, os autores concluem que, apesar de uma relativa menor resposta dos pacientes que não preenchem os critérios de inclusão dos ECRs ao tratamento com agentes biológicos, esses pacientes podem se beneficiar do seu uso. 
Genta MS, Kardes H, Gabay C: Clinical evaluation of a cohort of patients with rheumatoid arthritis treated with anti-TNFalpha in the community (Avaliação clínica de uma coorte com pacientes com artrite reumatóide tratados com anti-TNFalfa na comunidade). Joint Bone Spine 73(1): 51-6, 2006. Division of Rheumatology, Department of Internal Medicine, University Hospital of Geneva, Switzerland.

O objetivo deste estudo foi avaliar as respostas clínica, laboratorial e radiológica em uma coorte de pacientes com AR tratados com agentes anti-TNF na prática reumatológica. Os pacientes avaliados faziam parte de um banco de dados suíço de pacientes com AR (SCQM) provenientes de hospitais e clínicas privados. Todos os pacientes que iniciaram uso de agentes anti-TNF antes de fevereiro de 2003 foram incluídos; não se utilizou nenhum critério de exclusão. Os dados clínicos para avaliar a resposta terapêutica [índice de atividade de doença (DAS), questionário de qualidade de vida (HAQ) e escala visual analógica de dor (VAS)] e laboratoriais foram coletados antes do início do anti-TNF, aos 6 meses, 12 meses e após 20 meses de tratamento. Um total de 66 pacientes (média de idade de 60,5 anos), com doença de longa duração (média de 12,5 anos), foi analisado. $\mathrm{O}$ anti-TNF mais utilizado foi o etanercepte $(58 \%)$, seguido por infliximabe $(39 \%)$ e adalimumabe (3\%). Antes do início do anti-TNF, todos os pacientes haviam recebido em média quatro DMARDs diferentes. Dezesseis pacientes (24\%) descontinuaram o agente anti-TNF, a metade desses nos primeiros 6 meses do estudo. Após 6 meses, a média do DAS diminuiu de 4,8 $\pm 0,4$ para 3,8 $\pm 0,4(p<0,01)$; o HAQ permaneceu inalterado; e a dor, avaliada pela EVA, diminuiu de 5,0 para 3,3 em média $(\mathrm{p}<0,001)$. Esses índices permaneceram estáveis nas avaliações realizadas aos 12 meses e com mais de 20 meses. Os níveis séricos da proteína C-reativa diminuíram de forma gradativa de $17,9 \mathrm{mg} / \mathrm{dl}$ na linha de base para $5,6 \mathrm{mg} / \mathrm{dl}$ aos 20 meses ou mais. Nos pacientes em que foi possível obter dados radiológicos $(n=43)$, observaram-se diminuição na progressão de alterações radiológicas em 30 pacientes, piora radiológica em 12 e em 1 paciente o quadro radiológico permaneceu estável. Utilizando-se o DAS como parâmetro, 35 cinco pacientes (61\%) foram considerados respondedores ao tratamento aos 6 meses de acompanhamento. A resposta do tratamento com anti-TNF nesse pequeno grupo de pacientes foi similar aos resultados descritos em ensaios clínicos controlados.

Genevay S, Finckh A, Ciurea A, Chamot AM, Kyburz D, Gabay C; Physicians of the Swiss Clinical Quality Management Program for Rheumatoid Arthritis: Tolerance and effectiveness of anti-tumor necrosis factor alpha therapies in elderly patients with rheumatoid arthritis: a population-based cohort study (Tolerância e efetividade do tratamento com antifator de necrose tumoral alfa em pacientes idosos com artrite reumatóide: estudo de coorte populacional). Arthritis Rheum 57(4): 679-85, 2007. University Hospitals of Geneva, Geneva, Switzerland.

Este estudo publicado recentemente teve como objetivo avaliar a tolerância e efetividade dos agentes anti-TNF em pacientes idosos ( $\geq 65$ anos de idade) em comparação com pacientes mais jovens. As informações foram obtidas a partir do programa suíço SCQM-RA, o mesmo utilizado no artigo anterior, só que com dados mais atuais. O programa SCQM-RA é um estudo de coorte populacional multicêntrico para pacientes com AR. As autoridades regulatórias suíças recomendam a inclusão no programa de todos os pacientes que estejam recebendo agentes biológicos. Todos os pacientes do programa que receberam pelo menos uma dose de um agente anti-TNF no período de janeiro de 1997 a novembro de 2005 foram incluídos na análise. A tolerância foi avaliada pelo índice de descontinuação do medicamento e a efetividade, pelo DAS28 e HAQ. Entre os 1.571 pacientes com AR tratados com agentes anti-TNF, 344 tinham idade $\geq 65$ anos no início do tratamento e $1.227,<65$ anos. O tempo médio de seguimento foi de $22 \pm$ 15 meses. As taxas de descontinuação das drogas foram iguais entre os pacientes com idade $\geq 65$ anos e os pacientes com $<65$ anos (tempo médio de uso de 3,08 versus 3,04 anos). O principal motivo para a descontinuação do agente anti-TNF nos dois grupos foi falta de eficácia, seguida por reações alérgicas. Os valores de DAS28 diminuíram significativamente após 6 meses do início do agente anti-TNF e essa melhora foi similar entre os pacientes mais velhos e os mais jovens. A melhora no DAS28 se manteve com 2 anos de tratamento e permaneceu semelhante entre os dois grupos (média de - 0,65 versus - 0,58 ). Já o HAQ melhorou de forma menos significativa no grupo de pacientes com idade $\geq 65$ anos $(-0,02)$ que nos pacientes com $<65$ anos $(0,1)$; uma análise mais detalhada mostrou que esse achado se deveu basicamente aos índices de HAQ obtidos nos pacientes com $>$ de 75 anos de idade. Na discussão, os autores 
comentam que informações a respeito da eficácia e tolerabilidade de agentes biológicos e de outros tratamentos para AR são escassas na literatura, pois esses pacientes geralmente não são incluídos em ensaios clínicos. Os achados mais interessantes indicaram que a taxa de descontinuação e o índice de atividade da doença (avaliado pelo DAS28) após a introdução do agente
anti-TNF foram similares em pacientes com idade $\geq 65$ anos em comparação com os mais jovens. Os autores concluem que a idade não deve ser um fator que interfira na decisão de se iniciar um agente anti-TNF para o tratamento da AR. No subgrupo de pacientes com mais de 75 anos de idade, uma melhora funcional, avaliada pelo HAQ, não deve ser esperada.

Kristensen LA, Saxne T, Nilson JA, Geborek P: Impact of concomitant DMARD therapy on adberence to treatment with etanercepte and infliximabe in rheumatoid arthritis. Results from a six-year observational study in southern Sweden (Impacto do uso concomitante de DMARDs na aderência ao tratamento com etanercepte e infliximabe na artrite reumatóide. Resultado de um estudo observacional com seis anos de duração). Arthritis Res Ther 8: 1-10, 2006. Lund University Hospital, Lund, Sweden.

Neste estudo, foram incluídos pacientes do sul da Suécia com artrite reumatóide diagnosticados de acordo com o julgamento clínico de seu médico e que não haviam respondido ao uso de pelo menos dois DMARDs, incluindo o metotrexato (MTX), e estavam iniciando o uso de etanercepte ou de infliximabe pela primeira vez. No período de março de 1999 a dezembro de 2004, foram acompanhados 1.161 pacientes de acordo com protocolo clínico elaborado pelo South Swedish Arthritis Treatment Group (SSATG). A aderência ao tratamento em 1 e em 5 anos foi de $69 \%$ e $36 \%$ para o infliximabe e de $89 \%$ e $65 \%$ para pacientes tratados com etanercepte $(\mathrm{p}<0,001)$, respectivamente. Modelo de regressão de Cox demonstrou risco três vezes maior de interrupção precoce de tratamento nos pacientes que utilizaram infliximabe, em relação àqueles com etanercepte. Pacientes que utilizaram MTX concomitantemente ao anti-TNF apresentaram maior aderência ao tratamento em relação àqueles que utilizaram anti-TNF como monoterapia $(\mathrm{p}<0,001)$. Além disso, houve mais aderência ao uso de MTX como DMARD associado à terapia anti-TNF do que a outros DMARDs $(p<0,001)$. O sucesso com o uso do MTX foi devido à menor incidência de eventos adversos. Fatores de risco para suspensão precoce do tratamento foram baixos níveis de proteína C reativa (PCR), idade avançada, maior pontuação do HAQ e maior número de DMARDs utilizados previamente. Os autores concluíram que houve maior continuidade de tratamento com o uso de etanercepte do que de infliximabe e que a associação de MTX com anti-TNF melhora a aderência ao tratamento.

Kay JL, Griffiths ID for the BSR Biologics Register Management Committee: UK consultant rheumatologist's access to biological agents and views on the BSR Biologics Register (Acesso dos reumatologistas do Reino Unido a agentes biológicos e visão do Registro de Biológicos BSR). Rheumatology 45: 1376-9, 2006. Freeman Hospital and Newcastle University, Newcastle upon-Tyne, UK.

O British Society for Rheumatology Biologics Register (BSRBR) é um banco de dados nacional, no qual são incluídos todos os pacientes que recebem terapia anti-TNF para AR no Reino Unido. Neste artigo, foram avaliadas as respostas a um questionário enviado a todos os reumatologistas membros da British Rheumatology Society (BSR) em janeiro de 2006, que continha questões sobre o número de pacientes tratados, seus diagnósticos, limitações no acesso a terapias, opinião sobre o registro atual BSRBR e necessidade de novos registros, conduta na investigação de tuberculose subclínica e uso das diretrizes da BSR. Apenas 49,5\% dos reumatologistas britânicos responderam aos questionários. Dificuldade de acesso aos agentes anti-TNF foi apontada por $46 \%$ dos médicos, e problemas financeiros foram a principal limitação (70\%), mesmo para aqueles pacientes com AR que preenchiam os critérios do National Institute for Health and Clinical Exellence (NICE) para receberem biológicos. Entre os reumatologistas consultados, 67\% também conseguiram prescrever biológicos para pacientes com espondilite anquilosante e artrite psoriática em algumas circunstâncias, 
mas em apenas $25 \%$ e $35 \%$ dos casos, respectivamente, os biológicos foram prescritos de acordo com as diretrizes da BSR. Mais da metade dos reumatologistas relatou dificuldades no procedimento para submissão de dados para o BSRBR, embora a maioria tenha uma boa impressão sobre o BSRBR e acredita que registros semelhantes são desejáveis ou essenciais para espondilite anquilosante, artrite psoriática e para o rituximabe. Os autores concluíram que o acesso à terapia anti-TNF não é uniforme no Reino Unido, mesmo para pacientes com AR e que obedecem aos critérios do NICE. Há mais dificuldade ainda no acesso de doenças que não têm aprovação para o uso de anti-TNF pelo NICE. Para os reumatologistas britânicos, incluir pacientes no BSRBRé trabalhoso, mas considerado necessário.

Dixon WG, Watson K, Lunt M, Hyrich KL, British Society for Rheumatology Biologics Register Control Centre Consortium, Silman AJ, Symmons DPM on behalf of the British Society for Rheumatology Biologics Register: Rates of serious infection, including site-specific and bacterial intracellular infection, in rheumatoid arthritis patients receiving anti-tumor necrosis factor therapy. Results from the British Society for Rheumatology Biologics Register (Freqüência de infecções graves, incluindo infecções em sítios específicos e por bactérias intracelulares, em pacientes com artrite reumatóide que utilizam terapia antifator de necrose tumoral. Resultados do British Society for Rheumatology Biologics Register). Arthritis Rheum 54: 2368-76, 2006. University of Manchester, Manchester, UK.

Este estudo teve o objetivo de verificar se a freqüência de infecções graves é maior em pacientes com artrite reumatóide (AR) em uso de terapia antifator de necrose tumoral (anti-TNF) comparado àqueles tratados apenas com DMARDs tradicionais. Foram avaliados, prospectivamente, 7.664 pacientes com AR em uso de anti-TNF (3.596 com etanercepte, 2.878 com infliximabe e 1.190 com adalimumabe) e 1.354 pacientes com AR tratados com DMARDs tradicionais, todos oriundos do British Society for Rheumatology Biologics Register (BSRBR). Infecção grave foi definida como toda infecção que tenha levado à internação, ao óbito ou ao uso de antibioticoterapia intravenosa. Observaram-se 525 infecções graves no grupo anti-TNF e 56 no grupo DMARDs, após um seguimento de 1,26 e 0,94 ano, respectivamente, em cada grupo. A freqüência de infecção foi maior no grupo
anti-TNF (53 eventos/1.000 indivíduos por ano) em relação ao grupo DMARDs (4l eventos/1.000 indivíduos por ano). A freqüência de infecção grave de pele e tecido celular subcutâneo foi maior no grupo anti-TNF. Não houve diferença em relação ao risco de infecção entre os três medicamentos anti-TNF. Não houve divergência entre os dois grupos quanto ao risco de infecção nos 30 dias próximos a procedimentos cirúrgicos. Observaram-se 19 infecções graves por bactérias intracelulares apenas no grupo anti-TNF, 10 por Mycobacterium tuberculosis, 2 por Legionella pneumophila, 3 por Listeria monocytogenes, 1 por Mycobacterium fortuitum e 3 por Salmonella. Os autores concluíram que em pacientes com AR em atividade, o uso de anti-TNF não se associou a aumento no risco de infecções graves, exceto para infecções de pele e tecido celular subcutâneo.

Hyrich KL, Lunt M, Watson KD, Symmons DPM, Silman AJ for the British Society for Rheumatology Biologics Register: Outcomes after switching from one anti-tumor necrosis factor-agent in patients with rheumatoid arthritis. Results from a Large UK National Cohort Study (Evolução clinica após a troca de medicação anti-TNF em pacientes com artrite reumatóide. Resultados de um grande estudo de coorte nacional do Reino Unido). Arthritis Rheum 56: 13-20, 2007. University of Manchester, Manchester, UK.

Este estudo teve o objetivo de avaliar o risco de recorrência de não-resposta e de eventos adversos em pacientes que trocaram o agente anti-TNF por um segundo antiTNF. Analisaram-se 6.739 pacientes com artrite reumatóide que estavam iniciando anti-TNF como participantes do estudo de coorte prospectivo do Registro Nacional do Reino Unido. Entre eles, adalimumabe foi iniciado em 876, etanercepte, em 2.826 e infliximabe, em 3.037 pacientes. Em uma média de 15 meses de seguimento, 841 pacientes suspenderam o uso de anti-TNF por ineficácia e 1.023 por 
toxicidade. Destes, 503 e 353, respectivamente, passaram a receber um outro medicamento anti-TNF. Em geral, ao final do período de seguimento, $73 \%$ dos pacientes que trocaram de anti-TNF mantiveram o uso da segunda droga. A troca de agentes anti-TNF por ineficácia aumentou o risco de substituição da segunda droga também por ineficácia em 2,7 vezes (intervalo de confiança de 95\%, de 2,1-3,4), mas não houve risco de substituição por ineficácia quando o motivo da primeira substituição foi toxicidade (risco de
1,1; IC de 95\%, 0,9-1,5). De forma semelhante, a substituição do agente anti-TNF por toxicidade se associou a aumento no risco de uma segunda substituição também por toxicidade em 2,3 vezes (IC de 95\%, 1,9-2,9), mas não por falta de eficácia (risco de 1,2; IC de 95\%, 0,8-1,6). Os autores concluem que aqueles pacientes que substituem o anti-TNF apresentam alta freqüência de substituição do segundo anti-TNF, normalmente pelo mesmo motivo de substituição do primeiro anti-TNF. 